\title{
A power law extrapolation - interpolation method for IBNR claims reserving
}

\author{
Werner Hürlimann \\ Swiss Mathematical Society, Fribourg, Switzerland
}

Email address:

whurlimann@bluewin.ch

To cite this article:

Werner Hürlimann. A Power Law Extrapolation - Interpolation Method for IBNR Claims Reserving. Science Journal of Applied Mathematics and Statistics. Vol. 3, No. 1, 2015, pp. 6-13. doi: 10.11648/j.sjams.20150301.12

\begin{abstract}
To calculate claims reserves more frequently than the usual yearly periods for which ultimate loss development factors are available, it is necessary to perform an extrapolation prior to the time marking the end of the first development year and an interpolation for each successive development year. A simple power law extrapolation - interpolation method is developed and illustrated for monthly and quarterly sub-periods.
\end{abstract}

Keywords: Claims Reserving, IBNR Reserve, Loss Development Factors, Interpolation, Extrapolation, Power Law

\section{Introduction}

Claims reserves are usually the largest single item on an insurance company's balance sheet. Very often reserve fluctuations significantly affect the company's solvency requirements and overall financial position. Any mismatch of reserves has a direct impact on net asset values. Moreover, capital adequacy and reserving adequacy are essentially two sides of the same coin. An insurer whose claims reserves are more than adequate does not need to maintain as much capital as an insurer whose reserves are less than adequate. Setting claims reserves accurately is a gigantic task, especially for a complex multi-line insurer. Reserving has a great impact on virtually everything an insurance company does, from setting prices to establishing solvency margins. Therefore, with the introduction of Solvency II and the new accounting standards for insurance IFRS 4, reserving best practices are more and more important.

By nature, claims reserves are uncertain. Essentially, they are estimates of how much the company will have to pay out in the future on incurred claims, whether or not they have been reported. In simple terms, claims reserves consist of three key elements:

- $\quad$ Case estimates or case reserves are amounts for claims that have been notified to the company but have not yet been fully settled.

- Incurred but not enough reported (IBNER) are allowances for any inadequacies in case reserves.

- $\quad$ Incurred but not reported (IBNR) are estimated amounts for claims that have not yet been notified to the company. Companies seldom distinguish between IBNR and IBNER, instead combining them into a single item, called here simply $I B N R$ reserve. In the following, reported claims means the sum of the actual paid claims and the case reserves.

The present note is organized as follows. Section 2 recalls how IBNR reserves are calculated using the standard Chain Ladder method. To report IBNR reserves more frequently than the usual yearly periods, it is necessary to perform an extrapolation prior to the end of the first year and an interpolation for each successive development year. A simple power law method is developed and illustrated for monthly and quarterly sub-periods in Section 3.

\section{Calculation of IBNR Reserves}

In practice, the calculation of IBNR reserves involves an actuary, either at the initial stage or as part of the audit process. IBNR claims reserving can be described as "squaring the triangle", that is making use of historic information on the development of paid or reported claims to make estimates about their future development (e.g. Boulter and Grubbs [1], Subotzky and Mazur [23]). For example, at the end of 2014, a company that has been writing a certain class of business since 2005 has 10 annual development points for claims on its 2005 book of business, nine development points for 2006 and one for 2014. A loss triangle can be created with either the reported claims or the paid claims in form of a partially completed table. The rows represent the accident years in which claims incurred and the columns represent the development periods. 
Table 1 below is an example of loss triangle. This triangle will form the upper left part of a square (hence the expression, squaring the triangle) and the information in the triangle can be used to fill in the lower right part of the square, which, together with assumptions about the length of the development tail (accident years going beyond 2003), will give an estimate of the ultimate incurred claims. The difference between the estimated ultimate claims and the claims paid to date is the claims reserve, and the difference between the claims reserve and the outstanding case reserves for reported claims is the IBNR reserve.

The topic of claims reserving is well established within actuarial mathematics. Among recent work, one finds a handbook by Radtke and Schmidt [14], an extensive bibliography by Schmidt [21], and Ph.D. theses by Salzmann [17] and Happ [5].

The most commonly used IBNR reserving techniques are the Chain Ladder and the Bornhuetter-Ferguson methods or an optimal combination of them called Credible IBNR method (e.g. Mack [11], Hürlimann [9], Gigante et al. [4]). The methods are deterministic in that they give a point estimate of ultimate claims rather than a range of estimates. Other reserving methods, such as Bootstrapping or the Gamma $I B N R$ method in Hürlimann [8], are stochastic in that they use runoff triangles to arrive at a distribution of the ultimate claims (see also Wüthrich and Merz [24], Huang and Wu [6]). The statistical estimation of loss development factors in Table
2 is based on the data of Table 1 and uses for simplicity the Chain Ladder method.

In the Chain Ladder method, historical data is examined to estimate loss development factors (LDF) or ratios for each development period. The factors are cumulated and applied to the latest observed numbers (here paid claims) to estimate the ultimate incurred claims. The underlying assumption is that for each year of exposure, a certain percentage of the ultimate claims will have emerged at the end of each development year, and these percentages are consistent across years. So, for example, in Table 1, we can estimate the likely development of 2003 after five years by reference to the actual development of 1994 at 1999, 1995 at 2000, and so on.

For the mathematical specification, consider now a given accident year of a line of business over a development period $(0, T]$ in units of years. The ultimate LDF of the yearly exposure period $(t-1, t], t=1,2, \ldots, T$, of the considered accident year is denoted by $F_{t}$ (blue line in Table 2 with $\mathrm{T}=10)$. The further notations are as follows:

$S_{t}$ : aggregate paid claims for the period $(0, t]$

$O S_{t}$ : outstanding case reserves for the period $(t, T]$

$I B N R_{t}:$ IBNR reserve for the period $(t, T]$

By definition one has the identity:

$$
I B N R_{t}=\left(F_{t}-1\right) \cdot S_{t}-O S_{t} .
$$

Table 1. Loss Triangle of Paid Claims ("A.M. Best" 2004 table for Private Passenger Auto Liability).

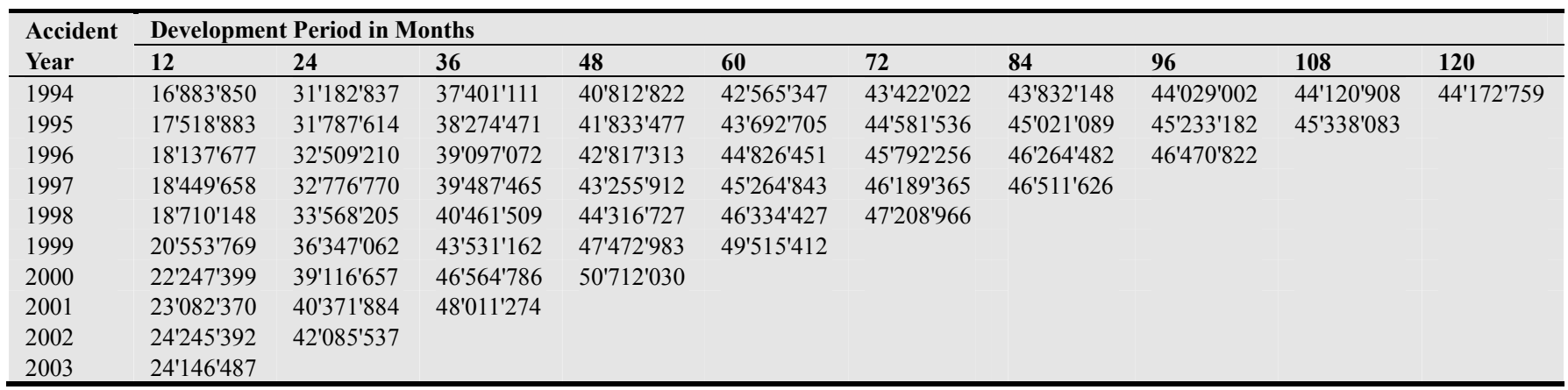

Table 2. Loss Development Factors according to the Chain Ladder method.

\begin{tabular}{|c|c|c|c|c|c|c|c|c|c|c|}
\hline Period in Months & $12-24$ & 24-36 & $36-48$ & $48-60$ & $60-72$ & $72-84$ & 84-96 & 96-108 & $108-120$ & 120-ult. \\
\hline Chain-ladder factors & 1.77805 & 1.19869 & 1.09270 & 1.04487 & 1.02025 & 1.00914 & 1.00455 & 1.00220 & 1.00118 & 1.00000 \\
\hline Ultimate LDF & 2.52532 & 1.42027 & 1.18485 & 1.08433 & 1.03776 & 1.01716 & 1.00795 & 1.00338 & 1.00118 & 1.00000 \\
\hline Percent Unpaid Claims & $60.40 \%$ & $29.59 \%$ & $15.60 \%$ & $7.78 \%$ & $3.64 \%$ & $1.69 \%$ & $0.79 \%$ & $0.34 \%$ & $0.12 \%$ & $0.00 \%$ \\
\hline Percent Paid Claims & $39.60 \%$ & $30.81 \%$ & $13.99 \%$ & $7.82 \%$ & $4.14 \%$ & $1.95 \%$ & $0.90 \%$ & $0.45 \%$ & $0.22 \%$ & $0.12 \%$ \\
\hline
\end{tabular}

\section{Extrapolation - Interpolation of Ultimate LDF Patterns}

To report IBNR reserves more frequently than the usual yearly periods for which ultimate LDF patterns are available, it is necessary to perform an extrapolation prior to time $t=1$ marking the end of the first year and an interpolation for each successive development year between time $t-1$ and time $t$. Different and more complex methods of extrapolation - interpolation have been developed earlier in Sherman [22] and Robbin and Homer [16].

For simplicity, let us focus on monthly and quarterly sub-periods, but the method is valid for sub-periods of arbitrary lengths. We assume that the revealed paid claims in each sub-period of a development year behave proportionally to a power law depending on the elapsed number of sub-periods as follows. Let the amounts of claims paid in the $k$-th sub-period of the development year $(t-1, t]$ equal $k^{\alpha} \cdot c_{t-1}^{(\alpha, m)}$ respectively $k^{\alpha} \cdot c_{t-1}^{(\alpha, q)}$, where $c_{t-1}^{(\alpha, m)}$ and $c_{t-1}^{(\alpha, q)}$ 
denote appropriate increment constants for the ultimate monthly respectively quarterly LDF patterns and $\alpha \in[0,1]$. The extreme case $\alpha=0$ refers to constant revealed paid claims in each sub-period and the extreme case $\alpha=1$ to a linear increase in the elapsed number of sub-periods. The Figures 1 and 2 yield a picture of this power law method. On the horizontal axis one finds the elapsed time and on the vertical axis the percentage of paid claims in a given development year.

The percentage of paid claims within a development year is highest (smallest) for $\alpha=0 \quad(\alpha=1)$. Other choices of the power law exponent $\alpha \in[0,1]$ lie between these extremes.

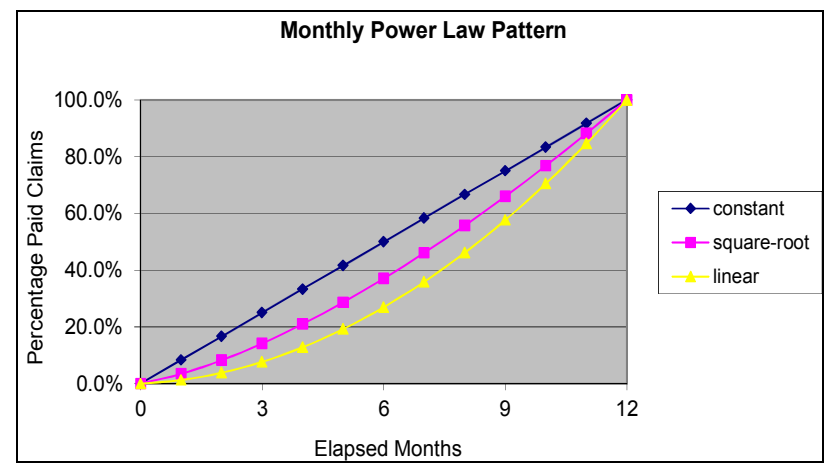

Figure 1. Monthly power-law extrapolation - interpolation pattern.

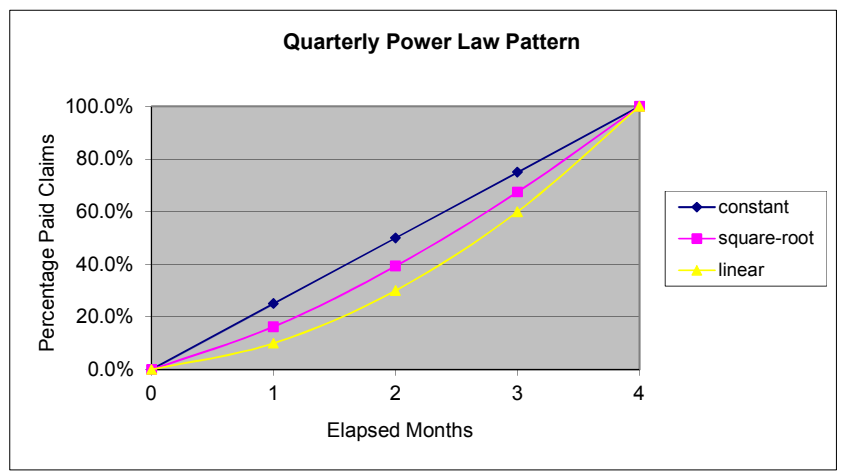

Figure 2. Quarterly power-law extrapolation - interpolation pattern.

The obtained ultimate monthly and quarterly LDF patterns after extrapolation and interpolation are elements of matrices denoted by

$F_{t-1, k}^{(\alpha, m)}$ : ultimate monthly LDF pattern for the $k$-th month of the development period $(t-1, t], t=1,2, \ldots, T, k=1,2, \ldots, 12$

$F_{t-1, k}^{(\alpha, q)}:$ ultimate quarterly LDF pattern for the $k$-th quarter of the development period $(t-1, t], t=1,2, \ldots, T, k=1,2,3,4$

To describe the obtained LDF patterns we will need the following quantities:

$U(t-1)$ : proportion of unpaid claims at time $t-1$ for the development period $(t-1, t], t=1,2, \ldots, T$

By definition of the ultimate yearly LDF pattern one has

$$
U(0)=1, \quad U(t-1)=1-\frac{1}{F_{t}}, \quad t=2, \ldots, T .
$$

A mathematical analysis yields the following formulas

$$
\begin{aligned}
c_{t-1}^{(\alpha, m)} & =\frac{U(t-1)-U(t)}{\sum_{k=1}^{12} k^{\alpha}}, \quad t=1,2, \ldots, T \\
F_{t-1,1}^{(\alpha, m)} & =\frac{1}{c_{t-1}^{(\alpha, m)}+1-U(t-1)}, \\
F_{t-1, k}^{(\alpha, m)} & =\frac{1}{k^{\alpha} \cdot c_{t-1}^{(\alpha, m)}+1 / F_{t-1, k-1}^{(\alpha, m)}}, \quad k=2, \ldots, 12 \\
c_{t-1}^{(\alpha, q)} & =\frac{U(t-1)-U(t)}{\sum_{k=1}^{4} k^{\alpha}}, \quad t=1,2, \ldots, T \\
F_{t-1,1}^{(\alpha, q)} & =\frac{1}{c_{t-1}^{(\alpha, q)}+1-U(t-1)}, \\
F_{t-1, k}^{(\alpha, q)} & =\frac{1}{k^{\alpha} \cdot c_{t-1}^{(\alpha, q)}+1 / F_{t-1, k-1}^{(\alpha, q)}}, \quad k=2,3,4
\end{aligned}
$$

A verification shows that at the extrapolating respectively interpolating times the formulas are consistent with the given ultimate yearly LDF pattern such that

$$
F_{t-1,12}^{(\alpha, m)}=F_{t-1,12}^{(\alpha, q)}=F_{t}, \quad t=1,2, \ldots, T .
$$

In practice one is also interested in the following quantities, where the symbol $\bullet$ stands for monthly $(m)$ or quarterly $(q)$ :

$U_{t-1, k}^{(\alpha, \bullet)}$ : proportion of unpaid claims at the end of the $k$-th sub-period of the development year $(t-1, t]$

$P_{t-1, k}^{(\alpha, \bullet)}$ : proportion of paid claims during the $k$-th sub-period of the development year $(t-1, t]$

$A P_{t-1, k}^{(\alpha, \bullet)}$ : proportion of aggregate paid claims at the end of the $k$-th sub-period of the development year $(t-1, t]$

These quantities are obtained using the following formulas:

$$
\begin{aligned}
U_{t-1, k}^{(\alpha, \bullet)} & =1-\frac{1}{F_{t-1, k}^{(\alpha, \bullet)}}, \\
U_{0,0}^{(\alpha, \bullet)} & =U(0)=1, \quad U_{t, 0}^{(\alpha, \bullet)}=U(t-1) \\
P_{t-1, k}^{(\alpha, \bullet)} & =U_{t-1, k-1}^{(\alpha, \bullet \bullet}-U_{t-1, k}^{(\alpha, \bullet)} \\
A P_{t-1, k}^{(\alpha, \bullet)} & =A P_{t-1, k-1}^{(\alpha, \bullet)}+P_{t-1, k}^{(\alpha, \bullet)} \\
A P_{0,0}^{(\alpha, \bullet)} & =0, \quad A P_{t, 0}^{(\alpha, \bullet)}=A P_{t-1,12}^{(\alpha, \bullet)}
\end{aligned}
$$

To illustrate, we have calculated the ultimate monthly and quarterly LDF patterns for the given ultimate yearly LDF pattern of Table 2 according to the above power law method 
for the linear case $\alpha=1$ (Tables 3 and 4), the constant case $\alpha=0$ (Tables 5 and 6) and the square root case $\alpha=\frac{1}{2}$ (Tables 7 and 8).

For comparison, percentages of unpaid claims in the sub-periods of the different development years have also been calculated for the linear case $\alpha=1$ (Tables 9 and 10), the constant case $\alpha=0$ (Tables 11 and 12) and the square root case $\alpha=\frac{1}{2}$ (Tables 13 and 14).

In the Tables 3 to 8 differences in numerical values of the various LDF patterns are observed for all calendar years. These are quite accentuated in the first year of development, which requires an extrapolation method. For the monthly LDF's they vary in the first month from 196.98 (linear case) and 73.86 (square root case) to 30.30 (constant case). The quarterly LDF's vary in the first quarter from 25.25 (linear case) and 15.52 (square root case) to 10.10 (constant case).

The percentages of unpaid claims (Tables 9 to 14) are less sensitive. For the monthly data, they vary in the first month from $99.5 \%$ (linear case) and $98.6 \%$ (square root case) to $96.7 \%$ (constant case). The quarterly percentages vary in the first quarter from 96\% (linear case) and 93.6\% (square root case) to $90.1 \%$ (constant case). These differences are significant enough to have a non-negligible impact on the reporting balance sheet of an insurance company. For example, given 100 Mio USD of expected ultimate claims, the maximum difference in unpaid reported claims can be as large as 5.9 Mio for claims reported in the first quarter of the first calendar year. Of course, the differences decrease with increasing calendar year because claims remaining unpaid diminish. However, in some lines of business, which can take many years to be fully developed, important differences will remain. To obtain a unique power law exponent $\alpha \in[0,1]$ an optimal criterion must be applied. This problem, which has not yet been investigated, is open for further investigation.

Table 3. Ultimate LDF Matrix by Year and Month (linear case).

\begin{tabular}{|c|c|c|c|c|c|c|c|c|c|c|c|c|c|c|}
\hline \multirow{2}{*}{ Year } & \multicolumn{12}{|l|}{ Month } & \multirow{2}{*}{$\begin{array}{l}\text { Yearly } \\
\text { Pattern }\end{array}$} & \multirow{2}{*}{$\begin{array}{l}\text { Increment } \\
\text { Constants }\end{array}$} \\
\hline & 1 & 2 & 3 & 4 & 5 & 6 & 7 & 8 & 9 & 10 & 11 & 12 & & \\
\hline 0 & 196.975 & 65.658 & 32.829 & 19.697 & 13.132 & 9.380 & 7.035 & 5.472 & 4.377 & 3.581 & 2.984 & 2.525 & 2.525 & $0.508 \%$ \\
\hline 1 & 2.500 & 2.452 & 2.383 & 2.296 & 2.197 & 2.088 & 1.974 & 1.858 & 1.743 & 1.631 & 1.523 & 1.420 & 1.420 & $0.395 \%$ \\
\hline 2 & 1.417 & 1.409 & 1.399 & 1.385 & 1.368 & 1.348 & 1.326 & 1.301 & 1.274 & 1.246 & 1.216 & 1.185 & 1.185 & $0.179 \%$ \\
\hline 3 & 1.183 & 1.181 & 1.176 & 1.171 & 1.164 & 1.156 & 1.147 & 1.136 & 1.125 & 1.112 & 1.099 & 1.084 & 1.084 & $0.100 \%$ \\
\hline 4 & 1.084 & 1.082 & 1.081 & 1.078 & 1.075 & 1.071 & 1.067 & 1.062 & 1.057 & 1.051 & 1.045 & 1.038 & 1.038 & $0.053 \%$ \\
\hline 6 & 1.017 & 1.017 & 1.016 & 1.016 & 1.015 & 1.015 & 1.014 & 1.013 & 1.012 & 1.011 & 1.009 & 1.008 & 1.008 & $0.012 \%$ \\
\hline 7 & 1.008 & 1.008 & 1.008 & 1.007 & 1.007 & 1.007 & 1.006 & 1.006 & 1.005 & 1.005 & 1.004 & 1.003 & 1.003 & $0.006 \%$ \\
\hline 8 & 1.003 & 1.003 & 1.003 & 1.003 & 1.003 & 1.003 & 1.003 & 1.002 & 1.002 & 1.002 & 1.002 & 1.001 & 1.001 & $0.003 \%$ \\
\hline 9 & 1.001 & 1.001 & 1.001 & 1.001 & 1.001 & 1.001 & 1.001 & 1.001 & 1.000 & 1.000 & 1.000 & 1.000 & 1.000 & $0.002 \%$ \\
\hline
\end{tabular}

Table 4. Ultimate LDF Matrix by Year and Quarter (linear case).

\begin{tabular}{|c|c|c|c|c|c|c|}
\hline \multirow{2}{*}{ Year } & \multicolumn{4}{|c|}{ Quarter } & \multirow{2}{*}{$\begin{array}{l}\text { Yearly } \\
\text { Pattern }\end{array}$} & \multirow{2}{*}{$\begin{array}{l}\text { Increment } \\
\text { Constants }\end{array}$} \\
\hline & 1 & 2 & 3 & 4 & & \\
\hline 0 & 25.253 & 8.418 & 4.209 & 2.525 & 2.525 & $3.960 \%$ \\
\hline 1 & 2.343 & 2.047 & 1.722 & 1.420 & 1.420 & $3.081 \%$ \\
\hline 2 & 1.393 & 1.340 & 1.269 & 1.185 & 1.185 & $1.399 \%$ \\
\hline 3 & 1.174 & 1.153 & 1.122 & 1.084 & 1.084 & $0.782 \%$ \\
\hline 4 & 1.079 & 1.070 & 1.056 & 1.038 & 1.038 & $0.414 \%$ \\
\hline 5 & 1.036 & 1.031 & 1.025 & 1.017 & 1.017 & $0.195 \%$ \\
\hline 6 & 1.016 & 1.014 & 1.012 & 1.008 & 1.008 & $0.090 \%$ \\
\hline 7 & 1.007 & 1.007 & 1.005 & 1.003 & 1.003 & $0.045 \%$ \\
\hline 8 & 1.003 & 1.003 & 1.002 & 1.001 & 1.001 & $0.022 \%$ \\
\hline 9 & 1.001 & 1.001 & 1.000 & 1.000 & 1.000 & $0.012 \%$ \\
\hline
\end{tabular}

Table 5. Ultimate LDF Matrix by Year and Month (constant case).

\begin{tabular}{|c|c|c|c|c|c|c|c|c|c|c|c|c|c|c|}
\hline \multirow{2}{*}{ Year } & \multicolumn{12}{|l|}{ Month } & \multirow{2}{*}{$\begin{array}{l}\text { Yearly } \\
\text { Pattern }\end{array}$} & \multirow{2}{*}{$\begin{array}{l}\text { Increment } \\
\text { Constants }\end{array}$} \\
\hline & 1 & 2 & 3 & 4 & 5 & 6 & 7 & 8 & 9 & 10 & 11 & 12 & & \\
\hline 0 & 30.304 & 15.152 & 10.101 & 7.576 & 6.061 & 5.051 & 4.329 & 3.788 & 3.367 & 3.030 & 2.755 & 2.525 & 2.525 & $3.300 \%$ \\
\hline 1 & 2.372 & 2.235 & 2.114 & 2.005 & 1.907 & 1.818 & 1.737 & 1.663 & 1.595 & 1.532 & 1.474 & 1.420 & 1.420 & $2.568 \%$ \\
\hline 2 & 1.397 & 1.375 & 1.353 & 1.332 & 1.312 & 1.292 & 1.273 & 1.254 & 1.236 & 1.219 & 1.201 & 1.185 & 1.185 & $1.166 \%$ \\
\hline 3 & 1.176 & 1.167 & 1.158 & 1.149 & 1.141 & 1.132 & 1.124 & 1.116 & 1.108 & 1.100 & 1.092 & 1.084 & 1.084 & $0.652 \%$ \\
\hline 4 & 1.080 & 1.076 & 1.072 & 1.068 & 1.064 & 1.061 & 1.057 & 1.053 & 1.049 & 1.045 & 1.041 & 1.038 & 1.038 & $0.345 \%$ \\
\hline 5 & 1.036 & 1.034 & 1.033 & 1.031 & 1.029 & 1.027 & 1.026 & 1.024 & 1.022 & 1.021 & 1.019 & 1.017 & 1.017 & $0.163 \%$ \\
\hline 6 & 1.016 & 1.016 & 1.015 & 1.014 & 1.013 & 1.013 & 1.012 & 1.011 & 1.010 & 1.009 & 1.009 & 1.008 & 1.008 & $0.075 \%$ \\
\hline 7 & 1.008 & 1.007 & 1.007 & 1.006 & 1.006 & 1.006 & 1.005 & 1.005 & 1.005 & 1.004 & 1.004 & 1.003 & 1.003 & $0.038 \%$ \\
\hline 8 & 1.003 & 1.003 & 1.003 & 1.003 & 1.002 & 1.002 & 1.002 & 1.002 & 1.002 & 1.002 & 1.001 & 1.001 & 1.001 & $0.018 \%$ \\
\hline 9 & 1.001 & 1.001 & 1.001 & 1.001 & 1.001 & 1.001 & 1.000 & 1.000 & 1.000 & 1.000 & 1.000 & 1.000 & 1.000 & $0.010 \%$ \\
\hline
\end{tabular}


Table 6. Ultimate LDF Matrix by Year and Quarter (constant case).

\begin{tabular}{lllllll}
\hline \multirow{2}{*}{ Year } & Quarter & & & \multicolumn{2}{c}{$\begin{array}{l}\text { Yearly } \\
\text { Pattern }\end{array}$} \\
\cline { 2 - 5 } & $\mathbf{1}$ & $\mathbf{2}$ & $\mathbf{3}$ & $\mathbf{4}$ & 2.525 \\
Constants
\end{tabular}

Table 7. Ultimate LDF Matrix by Year and Month (square root case).

\begin{tabular}{|c|c|c|c|c|c|c|c|c|c|c|c|c|c|c|}
\hline \multirow{2}{*}{ Year } & \multicolumn{12}{|l|}{ Month } & \multirow{2}{*}{$\begin{array}{l}\text { Yearly } \\
\text { Pattern }\end{array}$} & \multirow{2}{*}{$\begin{array}{l}\text { Increment } \\
\text { Constants }\end{array}$} \\
\hline & 1 & 2 & 3 & 4 & 5 & 6 & 7 & 8 & 9 & 10 & 11 & 12 & & \\
\hline 0 & 73.863 & 30.595 & 17.814 & 12.018 & 8.812 & 6.819 & 5.480 & 4.530 & 3.826 & 3.287 & 2.865 & 2.525 & 2.525 & $1.354 \%$ \\
\hline 1 & 2.460 & 2.373 & 2.274 & 2.170 & 2.065 & 1.960 & 1.859 & 1.761 & 1.668 & 1.581 & 1.498 & 1.420 & 1.420 & $1.053 \%$ \\
\hline 2 & 1.411 & 1.397 & 1.381 & 1.363 & 1.344 & 1.323 & 1.301 & 1.279 & 1.256 & 1.232 & 1.209 & 1.185 & 1.185 & $0.478 \%$ \\
\hline 3 & 1.181 & 1.176 & 1.169 & 1.162 & 1.154 & 1.146 & 1.136 & 1.127 & 1.117 & 1.106 & 1.095 & 1.084 & 1.084 & $0.267 \%$ \\
\hline 4 & 1.083 & 1.080 & 1.077 & 1.074 & 1.071 & 1.067 & 1.062 & 1.058 & 1.053 & 1.048 & 1.043 & 1.038 & 1.038 & $0.141 \%$ \\
\hline 5 & 1.037 & 1.036 & 1.035 & 1.033 & 1.032 & 1.030 & 1.028 & 1.026 & 1.024 & 1.022 & 1.020 & 1.017 & 1.017 & $0.067 \%$ \\
\hline 6 & 1.017 & 1.016 & 1.016 & 1.015 & 1.015 & 1.014 & 1.013 & 1.012 & 1.011 & 1.010 & 1.009 & 1.008 & 1.008 & $0.031 \%$ \\
\hline 7 & 1.008 & 1.008 & 1.007 & 1.007 & 1.007 & 1.006 & 1.006 & 1.005 & 1.005 & 1.004 & 1.004 & 1.003 & 1.003 & $0.015 \%$ \\
\hline 8 & 1.003 & 1.003 & 1.003 & 1.003 & 1.003 & 1.003 & 1.002 & 1.002 & 1.002 & 1.002 & 1.001 & 1.001 & 1.001 & $0.008 \%$ \\
\hline 9 & 1.001 & 1.001 & 1.001 & 1.001 & 1.001 & 1.001 & 1.001 & 1.001 & 1.000 & 1.000 & 1.000 & 1.000 & 1.000 & $0.004 \%$ \\
\hline
\end{tabular}

Table 8. Ultimate LDF Matrix by Year and Quarter (square root case).

\begin{tabular}{|c|c|c|c|c|c|c|}
\hline \multirow{2}{*}{ Year } & \multicolumn{4}{|c|}{ Quarter } & \multirow{2}{*}{$\begin{array}{l}\text { Yearly } \\
\text { Pattern }\end{array}$} & \multirow{2}{*}{$\begin{array}{l}\text { Increment } \\
\text { Constants }\end{array}$} \\
\hline & 1 & 2 & 3 & 4 & & \\
\hline 0 & 15.521 & 6.429 & 3.743 & 2.525 & 2.525 & $6.443 \%$ \\
\hline 1 & 2.242 & 1.934 & 1.656 & 1.420 & 1.420 & $5.013 \%$ \\
\hline 2 & 1.376 & 1.317 & 1.252 & 1.185 & 1.185 & $2.276 \%$ \\
\hline 3 & 1.167 & 1.143 & 1.115 & 1.084 & 1.084 & $1.273 \%$ \\
\hline 4 & 1.076 & 1.066 & 1.052 & 1.038 & 1.038 & $0.673 \%$ \\
\hline 5 & 1.034 & 1.030 & 1.024 & 1.017 & 1.017 & $0.318 \%$ \\
\hline 6 & 1.016 & 1.014 & 1.011 & 1.008 & 1.008 & $0.146 \%$ \\
\hline 7 & 1.007 & 1.006 & 1.005 & 1.003 & 1.003 & $0.074 \%$ \\
\hline 8 & 1.003 & 1.003 & 1.002 & 1.001 & 1.001 & $0.036 \%$ \\
\hline 9 & 1.001 & 1.001 & 1.000 & 1.000 & 1.000 & $0.019 \%$ \\
\hline
\end{tabular}

Table 9. Unpaid Claims Matrix by Year and Month (linear case).

\begin{tabular}{|c|c|c|c|c|c|c|c|c|c|c|c|c|c|}
\hline \multirow{2}{*}{ Year } & \multicolumn{12}{|l|}{ Month } & \multirow{2}{*}{$\begin{array}{l}\text { Yearly } \\
\text { Pattern }\end{array}$} \\
\hline & 1 & 2 & 3 & 4 & 5 & 6 & 7 & 8 & 9 & 10 & 11 & 12 & \\
\hline 0 & $99.5 \%$ & $98.5 \%$ & $97.0 \%$ & $94.9 \%$ & $92.4 \%$ & $89.3 \%$ & $85.8 \%$ & $81.7 \%$ & $77.2 \%$ & $72.1 \%$ & $66.5 \%$ & $60.4 \%$ & $60.4 \%$ \\
\hline 1 & $60.0 \%$ & $59.2 \%$ & $58.0 \%$ & $56.5 \%$ & $54.5 \%$ & $52.1 \%$ & $49.3 \%$ & $46.2 \%$ & $42.6 \%$ & $38.7 \%$ & $34.3 \%$ & $29.6 \%$ & $29.6 \%$ \\
\hline 2 & $29.4 \%$ & $29.1 \%$ & $28.5 \%$ & $27.8 \%$ & $26.9 \%$ & $25.8 \%$ & $24.6 \%$ & $23.1 \%$ & $21.5 \%$ & $19.7 \%$ & $17.8 \%$ & $15.6 \%$ & $15.6 \%$ \\
\hline 3 & $15.5 \%$ & $15.3 \%$ & $15.0 \%$ & $14.6 \%$ & $14.1 \%$ & $13.5 \%$ & $12.8 \%$ & $12.0 \%$ & $11.1 \%$ & $10.1 \%$ & $9.0 \%$ & $7.8 \%$ & $7.8 \%$ \\
\hline 4 & $7.7 \%$ & $7.6 \%$ & $7.5 \%$ & $7.2 \%$ & $7.0 \%$ & $6.7 \%$ & $6.3 \%$ & $5.9 \%$ & $5.4 \%$ & $4.9 \%$ & $4.3 \%$ & $3.6 \%$ & $3.6 \%$ \\
\hline 5 & $3.6 \%$ & $3.6 \%$ & $3.5 \%$ & $3.4 \%$ & $3.3 \%$ & $3.1 \%$ & $2.9 \%$ & $2.7 \%$ & $2.5 \%$ & $2.3 \%$ & $2.0 \%$ & $1.7 \%$ & $1.7 \%$ \\
\hline 6 & $1.7 \%$ & $1.7 \%$ & $1.6 \%$ & $1.6 \%$ & $1.5 \%$ & $1.4 \%$ & $1.4 \%$ & $1.3 \%$ & $1.2 \%$ & $1.1 \%$ & $0.9 \%$ & $0.8 \%$ & $0.8 \%$ \\
\hline 7 & $0.8 \%$ & $0.8 \%$ & $0.8 \%$ & $0.7 \%$ & $0.7 \%$ & $0.7 \%$ & $0.6 \%$ & $0.6 \%$ & $0.5 \%$ & $0.5 \%$ & $0.4 \%$ & $0.3 \%$ & $0.3 \%$ \\
\hline 8 & $0.3 \%$ & $0.3 \%$ & $0.3 \%$ & $0.3 \%$ & $0.3 \%$ & $0.3 \%$ & $0.3 \%$ & $0.2 \%$ & $0.2 \%$ & $0.2 \%$ & $0.2 \%$ & $0.1 \%$ & $0.1 \%$ \\
\hline 9 & $0.1 \%$ & $0.1 \%$ & $0.1 \%$ & $0.1 \%$ & $0.1 \%$ & $0.1 \%$ & $0.1 \%$ & $0.1 \%$ & $0.0 \%$ & $0.0 \%$ & $0.0 \%$ & $0.0 \%$ & $0.0 \%$ \\
\hline
\end{tabular}


Table 10. Unpaid Claims Matrix by Year and Quarter (linear case).

\begin{tabular}{|c|c|c|c|c|c|}
\hline \multirow{2}{*}{ Year } & \multicolumn{4}{|c|}{ Quarter } & \multirow{2}{*}{$\begin{array}{l}\text { Yearly } \\
\text { Pattern }\end{array}$} \\
\hline & 1 & 2 & 3 & 4 & \\
\hline 0 & $96.0 \%$ & $88.1 \%$ & $76.2 \%$ & $60.4 \%$ & $60.4 \%$ \\
\hline 1 & $57.3 \%$ & $51.2 \%$ & $41.9 \%$ & $29.6 \%$ & $29.6 \%$ \\
\hline 2 & $28.2 \%$ & $25.4 \%$ & $21.2 \%$ & $15.6 \%$ & $15.6 \%$ \\
\hline 3 & $14.8 \%$ & $13.3 \%$ & $10.9 \%$ & $7.8 \%$ & $7.8 \%$ \\
\hline 4 & $7.4 \%$ & $6.5 \%$ & $5.3 \%$ & $3.6 \%$ & $3.6 \%$ \\
\hline 5 & $3.4 \%$ & $3.1 \%$ & $2.5 \%$ & $1.7 \%$ & $1.7 \%$ \\
\hline 6 & $1.6 \%$ & $1.4 \%$ & $1.1 \%$ & $0.8 \%$ & $0.8 \%$ \\
\hline 7 & $0.7 \%$ & $0.7 \%$ & $0.5 \%$ & $0.3 \%$ & $0.3 \%$ \\
\hline 8 & $0.3 \%$ & $0.3 \%$ & $0.2 \%$ & $0.1 \%$ & $0.1 \%$ \\
\hline 9 & $0.1 \%$ & $0.1 \%$ & $0.0 \%$ & $0.0 \%$ & $0.0 \%$ \\
\hline
\end{tabular}

Table 11. Unpaid Claims Matrix by Year and Month (constant case).

\begin{tabular}{|c|c|c|c|c|c|c|c|c|c|c|c|c|c|}
\hline \multirow{2}{*}{ Year } & \multicolumn{12}{|l|}{ Month } & \multirow{2}{*}{$\begin{array}{l}\text { Yearly } \\
\text { Pattern }\end{array}$} \\
\hline & 1 & 2 & 3 & 4 & 5 & 6 & 7 & 8 & 9 & 10 & 11 & 12 & \\
\hline 0 & $96.7 \%$ & $93.4 \%$ & $90.1 \%$ & $86.8 \%$ & $83.5 \%$ & $80.2 \%$ & $76.9 \%$ & $73.6 \%$ & $70.3 \%$ & $67.0 \%$ & $63.7 \%$ & $60.4 \%$ & $60.4 \%$ \\
\hline 1 & $57.8 \%$ & $55.3 \%$ & $52.7 \%$ & $50.1 \%$ & $47.6 \%$ & $45.0 \%$ & $42.4 \%$ & $39.9 \%$ & $37.3 \%$ & $34.7 \%$ & $32.2 \%$ & $29.6 \%$ & $29.6 \%$ \\
\hline 2 & $28.4 \%$ & $27.3 \%$ & $26.1 \%$ & $24.9 \%$ & $23.8 \%$ & $22.6 \%$ & $21.4 \%$ & $20.3 \%$ & $19.1 \%$ & $17.9 \%$ & $16.8 \%$ & $15.6 \%$ & $15.6 \%$ \\
\hline 3 & $14.9 \%$ & $14.3 \%$ & $13.6 \%$ & $13.0 \%$ & $12.3 \%$ & $11.7 \%$ & $11.0 \%$ & $10.4 \%$ & $9.7 \%$ & $9.1 \%$ & $8.4 \%$ & $7.8 \%$ & $7.8 \%$ \\
\hline 4 & $7.4 \%$ & $7.1 \%$ & $6.7 \%$ & $6.4 \%$ & $6.1 \%$ & $5.7 \%$ & $5.4 \%$ & $5.0 \%$ & $4.7 \%$ & $4.3 \%$ & $4.0 \%$ & $3.6 \%$ & $3.6 \%$ \\
\hline 5 & $3.5 \%$ & $3.3 \%$ & $3.2 \%$ & $3.0 \%$ & $2.8 \%$ & $2.7 \%$ & $2.5 \%$ & $2.3 \%$ & $2.2 \%$ & $2.0 \%$ & $1.8 \%$ & $1.7 \%$ & $1.7 \%$ \\
\hline 6 & $1.6 \%$ & $1.5 \%$ & $1.5 \%$ & $1.4 \%$ & $1.3 \%$ & $1.2 \%$ & $1.2 \%$ & $1.1 \%$ & $1.0 \%$ & $0.9 \%$ & $0.9 \%$ & $0.8 \%$ & $0.8 \%$ \\
\hline 7 & $0.8 \%$ & $0.7 \%$ & $0.7 \%$ & $0.6 \%$ & $0.6 \%$ & $0.6 \%$ & $0.5 \%$ & $0.5 \%$ & $0.5 \%$ & $0.4 \%$ & $0.4 \%$ & $0.3 \%$ & $0.3 \%$ \\
\hline 8 & $0.3 \%$ & $0.3 \%$ & $0.3 \%$ & $0.3 \%$ & $0.2 \%$ & $0.2 \%$ & $0.2 \%$ & $0.2 \%$ & $0.2 \%$ & $0.2 \%$ & $0.1 \%$ & $0.1 \%$ & $0.1 \%$ \\
\hline 9 & $0.1 \%$ & $0.1 \%$ & $0.1 \%$ & $0.1 \%$ & $0.1 \%$ & $0.1 \%$ & $0.0 \%$ & $0.0 \%$ & $0.0 \%$ & $0.0 \%$ & $0.0 \%$ & $0.0 \%$ & $0.0 \%$ \\
\hline
\end{tabular}

Table 12. Unpaid Claims Matrix by Year and Quarter (constant case)

\begin{tabular}{|c|c|c|c|c|c|}
\hline \multirow{2}{*}{ Year } & \multicolumn{4}{|c|}{ Quarter } & \multirow{2}{*}{$\begin{array}{l}\text { Yearly } \\
\text { Pattern }\end{array}$} \\
\hline & 1 & 2 & 3 & 4 & \\
\hline 0 & $90.1 \%$ & $80.2 \%$ & $70.3 \%$ & $60.4 \%$ & $60.4 \%$ \\
\hline 1 & $52.7 \%$ & $45.0 \%$ & $37.3 \%$ & $29.6 \%$ & $29.6 \%$ \\
\hline 2 & $26.1 \%$ & $22.6 \%$ & $19.1 \%$ & $15.6 \%$ & $15.6 \%$ \\
\hline 3 & $13.6 \%$ & $11.7 \%$ & $9.7 \%$ & $7.8 \%$ & $7.8 \%$ \\
\hline 4 & $6.7 \%$ & $5.7 \%$ & $4.7 \%$ & $3.6 \%$ & $3.6 \%$ \\
\hline 5 & $3.2 \%$ & $2.7 \%$ & $2.2 \%$ & $1.7 \%$ & $1.7 \%$ \\
\hline 6 & $1.5 \%$ & $1.2 \%$ & $1.0 \%$ & $0.8 \%$ & $0.8 \%$ \\
\hline 7 & $0.7 \%$ & $0.6 \%$ & $0.5 \%$ & $0.3 \%$ & $0.3 \%$ \\
\hline 8 & $0.3 \%$ & $0.2 \%$ & $0.2 \%$ & $0.1 \%$ & $0.1 \%$ \\
\hline 9 & $0.1 \%$ & $0.1 \%$ & $0.0 \%$ & $0.0 \%$ & $0.0 \%$ \\
\hline
\end{tabular}

Table 13. Unpaid Claims Matrix by Year and Month (square root case).

\begin{tabular}{|c|c|c|c|c|c|c|c|c|c|c|c|c|c|}
\hline \multirow{2}{*}{ Year } & \multicolumn{12}{|l|}{ Month } & \multirow{2}{*}{$\begin{array}{l}\text { Yearly } \\
\text { Pattern }\end{array}$} \\
\hline & 1 & 2 & 3 & 4 & 5 & 6 & 7 & 8 & 9 & 10 & 11 & 12 & \\
\hline 0 & $98.6 \%$ & $96.7 \%$ & $94.4 \%$ & $91.7 \%$ & $88.7 \%$ & $85.3 \%$ & $81.8 \%$ & $77.9 \%$ & $73.9 \%$ & $69.6 \%$ & $65.1 \%$ & $60.4 \%$ & $60.4 \%$ \\
\hline 1 & $59.3 \%$ & $57.9 \%$ & $56.0 \%$ & $53.9 \%$ & $51.6 \%$ & $49.0 \%$ & $46.2 \%$ & $43.2 \%$ & $40.1 \%$ & $36.7 \%$ & $33.2 \%$ & $29.6 \%$ & $29.6 \%$ \\
\hline 2 & $29.1 \%$ & $28.4 \%$ & $27.6 \%$ & $26.7 \%$ & $25.6 \%$ & $24.4 \%$ & $23.1 \%$ & $21.8 \%$ & $20.4 \%$ & $18.8 \%$ & $17.3 \%$ & $15.6 \%$ & $15.6 \%$ \\
\hline 3 & $15.3 \%$ & $15.0 \%$ & $14.5 \%$ & $14.0 \%$ & $13.4 \%$ & $12.7 \%$ & $12.0 \%$ & $11.2 \%$ & $10.4 \%$ & $9.6 \%$ & $8.7 \%$ & $7.8 \%$ & $7.8 \%$ \\
\hline 4 & $7.6 \%$ & $7.4 \%$ & $7.2 \%$ & $6.9 \%$ & $6.6 \%$ & $6.2 \%$ & $5.9 \%$ & $5.5 \%$ & $5.0 \%$ & $4.6 \%$ & $4.1 \%$ & $3.6 \%$ & $3.6 \%$ \\
\hline 5 & $3.6 \%$ & $3.5 \%$ & $3.4 \%$ & $3.2 \%$ & $3.1 \%$ & $2.9 \%$ & $2.7 \%$ & $2.6 \%$ & $2.4 \%$ & $2.1 \%$ & $1.9 \%$ & $1.7 \%$ & $1.7 \%$ \\
\hline 6 & $1.7 \%$ & $1.6 \%$ & $1.6 \%$ & $1.5 \%$ & $1.4 \%$ & $1.4 \%$ & $1.3 \%$ & $1.2 \%$ & $1.1 \%$ & $1.0 \%$ & $0.9 \%$ & $0.8 \%$ & $0.8 \%$ \\
\hline 7 & $0.8 \%$ & $0.8 \%$ & $0.7 \%$ & $0.7 \%$ & $0.7 \%$ & $0.6 \%$ & $0.6 \%$ & $0.5 \%$ & $0.5 \%$ & $0.4 \%$ & $0.4 \%$ & $0.3 \%$ & $0.3 \%$ \\
\hline 8 & $0.3 \%$ & $0.3 \%$ & $0.3 \%$ & $0.3 \%$ & $0.3 \%$ & $0.3 \%$ & $0.2 \%$ & $0.2 \%$ & $0.2 \%$ & $0.2 \%$ & $0.1 \%$ & $0.1 \%$ & $0.1 \%$ \\
\hline 9 & $0.1 \%$ & $0.1 \%$ & $0.1 \%$ & $0.1 \%$ & $0.1 \%$ & $0.1 \%$ & $0.1 \%$ & $0.1 \%$ & $0.0 \%$ & $0.0 \%$ & $0.0 \%$ & $0.0 \%$ & $0.0 \%$ \\
\hline
\end{tabular}


Table 14. Unpaid Claims Matrix by Year and Quarter (square root case).

\begin{tabular}{|c|c|c|c|c|c|}
\hline \multirow{2}{*}{ Year } & \multicolumn{4}{|c|}{ Quarter } & \multirow{2}{*}{$\begin{array}{l}\text { Yearly } \\
\text { Pattern }\end{array}$} \\
\hline & 1 & 2 & 3 & 4 & \\
\hline 0 & $93.6 \%$ & $84.4 \%$ & $73.3 \%$ & $60.4 \%$ & $60.4 \%$ \\
\hline 1 & $55.4 \%$ & $48.3 \%$ & $39.6 \%$ & $29.6 \%$ & $29.6 \%$ \\
\hline 2 & $27.3 \%$ & $24.1 \%$ & $20.2 \%$ & $15.6 \%$ & $15.6 \%$ \\
\hline 3 & $14.3 \%$ & $12.5 \%$ & $10.3 \%$ & $7.8 \%$ & $7.8 \%$ \\
\hline 4 & $7.1 \%$ & $6.2 \%$ & $5.0 \%$ & $3.6 \%$ & $3.6 \%$ \\
\hline 5 & $3.3 \%$ & $2.9 \%$ & $2.3 \%$ & $1.7 \%$ & $1.7 \%$ \\
\hline 6 & $1.5 \%$ & $1.3 \%$ & $1.1 \%$ & $0.8 \%$ & $0.8 \%$ \\
\hline 7 & $0.7 \%$ & $0.6 \%$ & $0.5 \%$ & $0.3 \%$ & $0.3 \%$ \\
\hline 8 & $0.3 \%$ & $0.3 \%$ & $0.2 \%$ & $0.1 \%$ & $0.1 \%$ \\
\hline 9 & $0.1 \%$ & $0.1 \%$ & $0.0 \%$ & $0.0 \%$ & $0.0 \%$ \\
\hline
\end{tabular}

Let us conclude with a brief account of some related claims reserving literature and possible future developments.

Usually, claims reserving models assume independence between different accidents years. For this reason, they fail to model claims inflation appropriately, because claims inflation acts on all accident years simultaneously. A model that accounts for accident year dependence in runoff triangles has been proposed by Salzmann and Wüthrich [18].

Predictions of claims reserves often rely on individual loss triangles, where each triangle corresponds to a different line of business. Since different lines of business are often dependent it is necessary to develop models for loss triangle dependence. Examples that use copulas are Regis [15] and de Jong [2].

To take into account solvency purposes (e.g. the Solvency II project) it is necessary to adapt the classical claims reserving models. Some typical developments include Merz and Wüthrich [12], Hürlimann [7], Savelli and Clemente [19], Pira et al. [13], Eling et al. [3], Salzmann [17] and Happ [5].

Another direction concerns the development of claims reserving models based on multiple risk factors. Besides [7] and [20] we would like to point out [10], where the use of stochastic LDF's is advocated.

The integration of the presented simple extrapolation interpolation method in these and other recent claims reserving techniques and the study of its impact might be a topic for future research.

\section{References}

[1] A. Boulter and D. Grubbs, "Late claims in reinsurance", Publication Swiss Reinsurance Company, URL: http://www.swissre.com, 2000.

[2] P. De Jong, "Modeling dependence between loss triangles", North American Actuarial Journal 16(1), 74-86, 2012.

[3] M. Eling, D. Diers, M. Linde and C. Kraus, "The multi-year non-life insurance risk", Working papers on risk management and insurance no. 96, Institute of Insurance Economics, University of St. Gallen, November 2011.

[4] P. Gigante, L. Picech and L Sigalotti, "Prediction error for credible claims reserves: an h-likelihood approach", European Actuarial Journal 3(2), 453-470, 2013.
[5] S. Happ, "Stochastic claims reserving under consideration of various different sources of information", Dissertation, University Hamburg.

[6] Huang, J. and X. Wu, "Stochastic claims reserving in general insurance: models and methodologies", 2012 China International Conference on Insurance and Risk Management, Qingdao, 2012.

[7] W. Hürlimann, "Modelling non-life insurance risk for Solvency II in a reinsurance context", Life \& Pensions Magazine, January issue, 35-40, 2010.

[8] W. Hürlimann, "A Gamma IBNR claims reserving model with dependent development periods", Proc. $37^{\text {th }}$ Internat. ASTIN Colloquium, Orlando, 2007.

[9] W. Hürlimann, "Credible loss ratio claims reserves - the Benktander, Neuhaus and Mack methods revisited", ASTIN Bulletin 39(1), 81-100, 2009.

[10] W. Hürlimann, "Random loss development factor curves and stochastic claims reserving", JP Journal of Fundamental and Applied Statistics 1(1), 49-62, 2011.

[11] T. Mack, "Credible claims reserve: the Benktander method", ASTIN Bulletin 30(2), 333-347, 2000.

[12] M. Merz and M.V. Wüthrich, "Modelling the claims development result for solvency purposes", $38^{\text {th }}$ ASTIN Colloquium, Manchester, 2008.

[13] M. Pirra, S. Forte and M. Ialenti, "Implementing a Solvency II internal model : Bayesian stochasting reserving and parameter estimation", 40th ASTIN Colloquium, Madrid, 2011.

[14] M. Radtke and K.D. Schmidt, "Handbuch zur Schadenreservierung", 2nd ed., Verlag Versicherungswirtschaft, Karlsruhe, 2012.

[15] L. Regis, "A Bayesian copula model for stochastic claims reserving", working paper no. 227, Collegio Carlo Alberto, 2011.

[16] I. Robbin, D. Homer, "Analysis of loss development patterns using infinitely decomposable percent of ultimate curves", 1988 Discussion Papers on Evaluating Insurance Company Liabilities, Casual Actuarial Society, 503-538, URL: http://www.casact.org/pubs/dpp/dpp88/88dpp501.pdf

[17] R. Salzmann, "Stochastic claims reserving and solvency", Diss. ETH no. 20406, Zürich, 2012. 
[18] R. Salzmann, M.V. Wüthrich, "Modeling accounting year dependence in runoff triangles", European Actuarial Journal 2(2), 227-242, 2012.

[19] N. Savelli and G.P. Clemente, "Stochastic claims reserving based on CRM for Solvency II purposes", 40 ${ }^{\text {th }}$ ASTIN Colloquium, Madrid, 2011.

[20] M. Schiegl, "A three dimensional stochastic model for claim reserving”, preprint, arXiv: 1009.4146 [q-fin.RM], 2010.

[21] K.D. Schmidt, "A bibliography on loss reserving”, Technische Universität Dresden, update November 17, 2013.
[22] R.E. Sherman, "Extrapolating, smoothing and interpolating development factors", Proceedings of the Casualty Actuarial Society LXXI, 122-199, 1984.

[23] D. Subotzuky and J. Mazur, "How do you square the triangle", Reinsurance Magazine, Sept. 2006.

[24] M.V. Wüthrich and M. Merz, Stochastic Claims Reserving Methods in Insurance, J. Wiley, New York, 2008. 